Nouvelles perspectives en sciences sociales

\title{
L'intention est-elle si universelle que ne le prétendent les théories de l'action?
}

\section{Mélanie Girard, Simon Laflamme et Pascal Roggero}

Volume 1, numéro 2, mars 2006

URI : https://id.erudit.org/iderudit/602472ar

DOI : https://doi.org/10.7202/602472ar

Aller au sommaire du numéro

Éditeur(s)

Prise de parole

ISSN

1712-8307 (imprimé)

1918-7475 (numérique)

Découvrir la revue

Citer cet article

Girard, M., Laflamme, S. \& Roggero, P. (2006). L'intention est-elle si universelle que ne le prétendent les théories de l'action? Nouvelles perspectives en sciences sociales, 1(2), 115-148. https://doi.org/10.7202/602472ar d'utilisation que vous pouvez consulter en ligne. 


\title{
L'intention est-elle si universelle que ne le prétendent les théories de l'action?
}

\author{
MÉLANIE GIRARD \\ Université de Toulouse 1, CIRESS-LEREPS-GRES, France \\ SimOn LALFLAMmE \\ Université Laurentienne, Ontario, Canada \\ Pascal Roggero \\ Université de Toulouse 1, CIRESS-LEREPS-GRES, France
}

\section{Pour une vérification des affirmations des théories de l'action}

Il est impossible à l'homme de vivre en tant que tel en l'absence d'un univers symbolique, d'un langage, et donc d'un ensemble d'informations à la fois structuré et structurant - mais non pas fini--aussi bien que rationnel et non rationnel, sur lequel il peut lui-même intervenir. Grâce à ce langage, l'humain peut communiquer avec les autres et avec soi, interpréter son environnement et développer une psyché où cohabitent l'émotion et la raison, le logique et le non-logique. Il intériorise ainsi sa société en même temps qu'il la construit; par cette dialectique, il se fait, par essence, historique. Que l'humain soit social, historique et communicationnel, que son vécu soit complexe, ce sont là des évidences. On doit donc s'attendre à ce que toute sociologie de l'acteur l'assume et en tire les conséquences. Or, nous posons la question de savoir si c'est bel et bien ce que font ces sociologies. Et ces sociologies sont nombreuses, dominantes, bien qu'elles 
ne reconnaissent pas leur domination. Il y a, entre autres, le fonctionnalisme stratégique ${ }^{1}$, l'individualisme méthodologique ${ }^{2}$, la théorie du choix ration$n l^{3}$, la théorie de l'échange ${ }^{4}$, la théorie des réseaux ${ }^{5}$, le structuralisme génétique $^{6}$, l'ethnométhodologie $e^{7}$ l'analyse conversationnelle ${ }^{8}$, l'ethnographie institutionnelle ${ }^{9}$, le néofonctionnalisme ${ }^{10}$, l'actionalisme ${ }^{11}$, l'interactionisme symbolique ${ }^{12}$. Dans toutes ces approches, en effet, l'acteur social est bel et bien défini comme rationnel. Et cette caractéristique a constamment pour corollaire une série de qualités. Dans ces cadres théoriques, si l'acteur peut être déclaré comme rationnel, et de façon principielle, c'est aussi qu'il est simultanément compris comme intéressé, stratégique, conscient, réfléchi, autonome, intentionné. Parfois, il est vrai, cette sociologie aménage un certain espace à la dimension non rationnelle de l'humain. Mais ce scrupule ne modifie en rien l'affirmation de principe, et surtout pas le mode d'analyse qui est suggéré, lequel demeure centré sur un acteur perçu comme

'Voir, par exemple, Michel Crozier et Erhard Friedberg, L'acteur et le systeme, Paris, Seuil, Point politique, 1977.

${ }^{2}$ Voir, par exemple, Raymond Boudon, I a place du désordre. Critique des theories du changement, Paris, PUF, [1984] 1991.

${ }^{3}$ Debra Friedman et Michael Hechter, "The Contribution of Rational Choice Theory to Macrosociological Research », Sociological Theory, n"6, 1988, p. 201-218.

${ }^{4}$ Voir, par exemple, George C. Homans, Social Behavior: Its Elementary Forms, New York, Hartcourt, Brace and World, 1961 ; Peter Blau, Exchange and Poner in Social L iffe, New York, Wiley, 1964.

${ }^{5}$ Voir, par exemple, Harrison White, Identity and Control: A Structural Theory of Social Action, Princeton, N.J., Princeton University Press, 1992.

"Voir, par exemple, Pierre Bourdieu, 1 a distinction : critique sociale du jugement, Paris, Minuit, 1979.

${ }^{7}$ Voir, par exemple, Harold Garfinkel, Studies in Ethnomethodology, Englewood Cliffs, N.J., Prentice-Hall, 1967.

${ }^{8}$ Voir, par exemple, Harvey Sacks, Lectures on Conversation, Oxford, Blackwel, 1995.

"Voir, par exemple, Dorothy Smith, The Conceptual Practices of Power: A Feminist Sociology of Knowledge, Toronto, University of Toronto Press, 1990.

${ }^{10}$ Voir, par exemple, Jeffrcy Alexander, Theoretical Logic in Sociology, vol. 1-4, Berkeley, University of California Press, 1982-1983.

${ }^{11}$ Voir, par exemple, Alain Touraine, Le retour de l'acteur : essai de sociologie, Paris, Fayard, 1984 ou Critique de la modemité, Paris, Fayard, 1992.

12 Voir, par exemple, Erving Goffman, The Presentation of Self in Everyday I-ife, Woodstock, New York, Overlook Press, 1973. 
étant motivé par la raison. C'est le cas, par exemple, chez Weber, où, dans la typologie, figure l'action émotive, mais où toute la méthode oblige à comprendre l'acteur en tant qu'il agit dans un rapport entre des moyens et des fins. C'est le cas aussi, de Crozier et Friedberg ${ }^{13}$ qui notent qu'il y a souvent émotion chez les acteurs, mais qui n'en comprennent les faits et gestes que dans la mesure où ils sont articulés à une stratégie.

On peut, ici, s'étonner d'un discours aussi emphatique. On peut se demander pourquoi ces sociologies insistent pour n'appréhender l'acteur que sous l'angle de sa rationalité ; pourquoi elles occultent toute cette humanité qui est faite de paradoxes, d'émotions; pourquoi des phénomènes aussi notoires que l'amour entre conjoints, que la haine de l'autre, que la tendresse d'un parent envers son enfant, que la peine de l'être abandonné, que la satisfaction du vainqueur, que la confusion dans les idées n'apparaissent pas, à ces sociologies, dignes de s'inscrire dans un modèle d'analyse destiné à rendre intelligible l'agir humain. On peut encore se demander pourquoi il est utile que l'acteur social soit considéré comme autonome, alors que l'aliénation est une réalité et qu'on ne peut pas, si libre soit-on, ne pas être déterminé, si partiellement cela soit-il, par son milieu; pourquoi il faut rappeler l'idée d'un acteur stratégique quand la spontanéité, la routine ou l'impulsion sont si typiquement humaines; pourquoi il est nécessaire que l'acteur soit présenté comme n'agissant que de façon intéressée quand ses propres gestes lui sont souvent préjudiciables ; pourquoi il importe qu'on ne voie l'humain qu'en tant que conscient alors que chacun observe quotidiennement le fait de l'inconscience, que le rêve occupe un immense espace dans les psychés; pourquoi il faut que l'humanité soit définie par l'intention alors que la plupart des gestes que chacun pose dans une journée ne sont précédés d'aucune réflexion, alors que les paroles que tous prononcent relèvent moins de projets que de l'animation de dynamiques relationnelles qui ont lieu dans l'instantanéité. Et l'on peut, de surcroit, s'interroger à savoir pourquoi il faut mettre de l'avant la notion de subjectivité quand il n'y a, en réalité, de sujet que parce qu'il y a des relations humaines, quand il n'y a pas de subjectivité qui puisse se concevoir en dehors d'un rapport à l'autre et, plus généralement, àl'environnement, c'està-dire quand il n'y a pas de subjectivité extrasociale. Certes l'acteur social est capable de stratégie, d'autonomie, d'égotisme, de conscience, d'intention, de réflexion, mais il est aussi capable de bien d'autres choses. Or, ces autres choses, l'individualisme méthodologique ne les aperçoit pas, lui qui ne peut 
concevoir d'action que si elle est commandée par l'intérêt rationnellement interprété en termes de gains et de coûts et qui ne parvient à nuancer sa position qu'en faisant allusion à une rationalité limitée, laquelle rationalité n'est limitée que parce que des informations lui font défaut ou parce qu'elle est circonstancielle. La théorie du choix rationnel ou ses dérivés, la théorie de l'échange ou la théorie des réseaux, non plus ne les voient pas, elles pour lesquelles il n'existe même pas de façon autre d'être socialement que par évaluation des bénéfices et des pertes en toute chose. Il en va de même du structuralisme génétique pour qui tout acteur tente de préserver des privilèges de classe ou d'acquérir de tels privilèges et, donc, agit toujours en fonction de son intérêt. Il en va de même, aussi, de l'ethnométhodologie, dont l'essentiel du discours consiste à affirmer que l'acteur n'est pas déterminé par les structures sociales et qu'il ne peut en être ainsi parce qu'il est réfléchi, puis rationnel, puis autonome, puis conscient et ainsi de suite, dans une parfaite circularité. Il en va de même encore de l'analyse conversationnelle qui ne comprend l'agent communicant qu'en tant qu'il est constamment en train de solutionner des problèmes liés à la communication. Il en va toujours de même de l'ethnographie institutionnelle qui veut que tout propos se comprenne par identification de la position sociale (standpoint) de son auteur et qui réduit ainsi ce qui peut être dit à l'intérêt qui découle de l'appartenance à un groupe. Il en va toujours et encore de même du néofonctionnalisme pour lequel l'action est seconde par rapport à la structure, cependant que cette action est déclarée comme rationnelle et qu'elle est telle parce qu'elle est toujours la conséquence d'une réflexion sur les profits et les pertes. Il en va toujours et toujours de même de l'actionnalisme, surtout dans sa deuxième phase, où l'on assiste à une ode aux qualités de cet acteur qui est rationnel, réfléchi, conscient... Il en va, enfin, pareillement de l'interactionnisme symbolique, qui, bien qu'il ait compris que l'acteur est nécessairement en relation, ne dépeint jamais les rapports sociaux qu'à partir d'un acteur dont le « rôle » consiste rationnellement à présenter aux autres une image de soi favorable.

L'approche rationalisante n'est pas exclusive. Il est, en effet, on le sait, des sociologies, si critiquables soient-elles, dont le point de mire n'est pas l'acteur et qui parviennent, néanmoins, à rendre intelligibles des réalités humaines. Qu'on pense au fonctionnalisme ${ }^{14}$ qui attire l'attention sur ce qui, à l'extérieur de l'individu, rend probable son comportement. Qu'on pense

${ }^{14}$ Émile Durkheim, Les règles de la méthode sociologique, Paris, PUF, [1894] 1937 ou Le suicide : étude de sociologie, Paris, PUF, [1897] 1969. 


\section{MÉLANIE GIRARD ... / LINTENTION EST-ELLE SI UNIVERSELLE ...?}

au structuralisme foucaldien ${ }^{15}$ qui met en lumière un ensemble de conditions institutionnelles qui rendent possible un ou des discours à un moment spécifique de l'histoire. Qu'on pense aussi à la théorie des systèmes sociaux ${ }^{16}$ pour laquelle la subjectivité n'est elle-même qu'un système parmi d'autres. L'approche rationalisante a donc son autre. De surcroit, elle éveille parfois la suspicion. C'est ainsi, par exemple, que l'antiutilitarisme ${ }^{17}$ constate que l'explication de l'action humaine par stricte évocation d'une raison intéressée coupe d'une infinité de pensées et de gestes humains. C'est ainsi encore que la théorie de l'agir communicationnel ${ }^{18}$ voudrait sortir des couloirs qui conduisent nécessairement vers la subjectivité et propose de se tourner vers un acteur non pas intelligible en lui-même, mais en fonction de la relation qu'il entretient avec les autres ; cette théorie parle alors beaucoup plus d'intercompréhension - catégorie quasi relationnelle - que de compréhension - catégorie plutôt phénoménologique. Dans le cas de l'antiutilitarisme, toutefois, le doute n'a pour fin que de substituer la générosité humaine à l'égoïsme, dont l'intérêt est le corollaire, et l'approche continue de se nourrir des catégories phénoménologiques, notamment de l'intention. Dans le cas de la théorie de l'agir communicationnel, la solution qui est proposée, pour rendre compte de l'entente à laquelle parviennent les agents communicants, convoque le rationalisme intentionnel, redonne du service à une raison transcendante et crée alors une espèce de néophénoménologie qui dérelativise, déshistoricise les relations humaines. On trouve d'autres remises en question de l'appareil conceptuel de l'analyse rationalisante. Louis Quéré, par exemple, observe, lui aussi, que l'analyse de l'action se déploie autour d'un vocabulaire spécifique, d'un « système conceptuel » qui « comporte des notions telles que motif, intention, désir, but, délibération, volonté, contraintes, résultats voulus, etc. ». Ces notions, juge-t-il, sont

${ }^{15}$ Michel Foucault, Les mots et les choses. Une archéologie des sciences humaines, Paris, Gallimard, Bibliothèque des sciences humaines, 1966 ou L'arcbéologie du savoir, Paris, Gallimard, Bibliothèque des sciences sociales, 1969.

${ }^{16}$ Niklas Luhmann, Social Systems, Stanford, Cal., Stanford University Press, [1984] 1995.

${ }^{17}$ Jacques T. Godbout et Alain Caillé, L'esprit du don, Montréal, Boréal / Paris, La Découverte, 1992; Alain Caillé, Critique de la raison utilitaire. Manifeste du MAUSS, Paris, La Découverte, 1989 ou Don, intérêt et désintéressement. Bourdieu, Mauss, Platon et quelques autres, Paris, La Découverte / M.A.U.S.S., 1994.

${ }^{18}$ Jürgen Habermas, Théorie de l'agir communicationnel, tomes I et II, Paris, Fayard, L'espace du politique, [1981] 1987 ou Le discours philosophique de la modernité. Douze conférences, Paris, Gallimard, Bibliothèque de philosophie, [1985] 1988. 
organisées en un réseau et " s'entre-signifient »; le recours à ce corpus permet de " configurer l'action en réponse à un questionnement spécifique » qui « se fait dans des formes d'attribution, de description et d'explication appropriées au caractère intentionnel de l'action $»{ }^{19}$ Dénonçant cette approche surrationalisante, Quéré invite son lecteur à se rappeler qu'il existe des formes «pré-intentionnelles » ou « infra-intentionnelles » de l'action humaine.

Les sociologies rationalisantes ont beaucoup en commun. Elles ont toutes une orientation phénoménologique en ce qu'on y trouve l'omniprésence de la catégorie d'intention. Jean-Michel Berthelot arrive à la même conclusion en parlant des théories de l'action :

\begin{abstract}
Ainsi, on peut montrer que les différentes théories de l'action [...] (utilitarisme, phénoménologie, ethnométhodologie, interactionnisme symbolique...) ne sont pas aussi éloignées qu'il y parait à première vue. Elles se regroupent, en effet, malgré leur déni réciproque, sous un schème commun : celui de l'intentionnalité. Selon ce schème, un phénomène social donné (une décision d'investissement, une évolution de la consommation, le développement imprévisible d'une manifestation, etc.) résulte de la combinaison des intentions d'un ensemble d'acteurs inscrits dans un système d'action. ${ }^{20}$
\end{abstract}

Dans l'optique de ces sociologies, il y a humanité parce qu'il y a intention - et donc conscience, raison, intérêt... Mais la question se pose de savoir si l'agir humain est si nécessairement, si universellement intentionnel. On notera au passage que l'approche phénoménologique ne conçoit à cet égard aucune variation selon quelque référent exogène que ce soit. L'humain, toujours et partout, parce qu'il est humain, fait ou dit quelque chose parce qu'il en a préalablement l'intention. Même les relations ne sont aucunement perçues comme génératrices des gestes et des propos puisque l'acteur doit toujours avoir la capacité d'agir intentionnellement. On peut alors se demander si, en mettant ainsi l'accent sur la subjectivité, on ne s'empêche pas de comprendre l'humain dans les relations qu'il entretient avec les autres, avec le monde, relations sans lesquelles, par ailleurs, il ne

" Louis Quéré, "Langage de l'action et questionnement sociologique », in Paul Ladrière, Patrick Pharo et Louis Quéré (dir.), La théorie de l'action. Le sujet pratique en débat, Paris, CNRS, 1993, p. 57-58.

20) Jean-Michel Berthelot, «Le devoir d'inventaire », in Philippe Cabin (dir.), La sociologie. Histoires et idées, Auxerre, Sciences humaines, 2000, p. 250-251. 
peut y avoir de conscience même. C'est en tout cas l'interrogation à laquelle convie une approche plus relationnelle ${ }^{21}$.

Une approche relationnelle propose que l'analyse porte davantage sur la relation que sur l'action puisque, de toute façon, il n'y a d'action ou de discours que dans et par les relations. Mais ce déplacement n'est pas sans conséquences. Il a pour fin d'imposer à l'analyse la pleine reconnaissance du caractère essentiellement social et historique de l'humain et d'obliger à ne pas réduire l'humanité à sa rationalitée ${ }^{22}$, de lui conférer, donc, un peu de cette complexité dont on fait mention dans les réflexions moriniennes ${ }^{23}$. Il y a moins humanité parce qu'il y a subjectivité que parce qu'il y a relation humaine. Il y a humanité parce que la relation humaine est communication, que cette communication est symbolique et que ces symboliques sont marquées de l'historicité autant que de la socialité, que, dans cette socialité et cette historicité, l'humain détermine autant l'ordre du communicable qu'il est déterminé par lui. Poser l'acteur comme relationnel, c'est, du même coup, affirmer qu'il est social, puisque les relations se définissent dans et par la socialité, celle-ci renfermant les éléments de la communication nécessaires à la reproduction ou à la transformation de relations spécifiques. Aborder d'emblée l'humain en tant qu'agent communicant, et donc dans son rapport à des symboliques, c'est le concevoir à travers un langage porteur de rationalité autant que d'émotion puisque les codes humains contraignent à une certaine logicité, sans qu'ils enferment en elle ni l'histoire, ni la socialité, et puisqu'ils rendent possible l'expression de ce qui est éprouvé dans le vécu de chacun et de plusieurs, sensation beaucoup plus complexe que ne peut la concevoir une approche rationalisante. Dire que l'humain est relationnel, c'est affirmer que, dans sa quotidienneté, il se situe au carrefour de l'émotion et de la raison puisque, en aucun cas, la rationalité ne pourrait contenir et générer ce qui peut être vécu. Dans l'optique d'une approche relationnelle, l'acteur social en tant que tel est toujours non pas rationnel mais « émorationnel », c'est-à-dire qu'il est à la fois raison et émotion.

${ }^{21}$ Simon Laflamme, Communication et émotion. Essai de microsociologie relationnelle, Paris, L.Harmattan, 1995.

${ }^{22}$ Mélanie Girard, Relations bumaines et production d'information : l'échange comme objet d'étude d'une approche relationnelle, mémoire de maitrise, Sudbury, Université Laurentienne, 2004 et Pierre Bouchard, Contribution à la critique de la rationalité utilitaire. Pour un modèle de remplacement des théories de l'action bumaine, mémoire de maitrise, Sudbury, Université Laurentienne, 2000.

${ }^{23}$ Edgar Morin, La méthode, tomes 1-6, Paris, Seuil, 1977-2004. 
Le recours à la notion d'historicité a pour fin de rappeler que l'humain est toujours en train de se faire, qu'il détermine son destin autant qu'il est déterminé par son milieu, qu'il est inscrit dans une histoire individuelle aussi bien que collective. L'historicité humaine est à la fois spécifique et non spécifique. Dans une relation, quelque chose de particulier advient; cette relation a donc une histoire originale. Mais cette relation s'insère elle-même dans une dynamique qui la dépasse puisque des modes relationnels se conjuguent, puisqu'ils présentent des similitudes, recourent à des symboliques et à des codes qui ne sont pas exclusifs, puisqu'y interviennent des valeurs, des normes, des principes qui agissent à une échelle étendue.

Le rappel de la notion de socialité a pour objectif de souligner l'impossibilité pour l'humain de vivre dans le pur isolement, de ne pas communiquer, d'être en dehors du langage. L'humain pense, éprouve largement avec les mots, et donc dans un univers communicationnel, dans la socialité. Le langage est par essence social. L'humain est par essence socialisé.

La contraction, dans le terme émoraison, des mots émotion et raison, rappelle que les échanges entre les agents communicants sont toujours faits, dans le champ de l'action sociale en tant que telle, à la fois d'émotion et de raison et qu'il est impossible d'isoler tout à fait l'une ou l'autre de ces deux dimensions. Un échange en apparence strictement émotif, par exemple, comportera forcément une certaine logique, sera constitué de raisonnements. Inversement, un échange dont le discours est rigoureux contiendra des aspects émotifs; tout cela dans une intrication telle que les deux dimensions tissent les propos autant que les psychés sans jamais pouvoir s'anéantir l'une l'autre, en intervenant en même temps dans des combinaisons, dans des confusions qui font en sorte que l'humain est en mesure de faire intervenir l'ordre du senti dans celui de la science et de produire ainsi un vécu où agissent les uns sur les autres, dans des ensembles infiniment riches, le rêve, l'affection, la perspicacité, la mémoire, la projection, le savoir, la critique, l'imagination ${ }^{24} \ldots$ Dans l'Homo sapiens, écrit Morin, il faut aussi voir un Homo demens ${ }^{25}$.

\footnotetext{
${ }^{24}$ Et c'est parce qu'il en est ainsi que le travail scientifique consiste largement en une désubjectivation.

${ }^{25}$ Edgar Morin, Le paradigme perdu, la nature bumaine, Paris, Seuil, 1973.
} 
L'humain est un être historique, social, rationnel, émotif, relationnel, communicationnel. Il n'y a rien d'original à l'affirmer ; ce sont là quelquesunes des grandes conclusions de la sociologie du $\mathrm{XX}^{\mathrm{e}}$ e siècle. On trouve d'ailleurs, dans bon nombre de théories, une ou plusieurs de ces affirmations. Mais il y a un problème : on ne les trouve qu'exceptionnellement réunies dans une même théorie alors qu'elles ne sont pas mutuellement exclusives ; c'est aussi que, dans la plupart des cadres théoriques, l'exploitation de l'une d'elles en élimine d'autres : l'approche rationalisante repousse l'émotion, l'attention qui est portée sur l'acteur empêche d'aménager quelque espace à la relation et davantage à la communication. Or, il nous semble qu'une théorie générale du social doit au moins être en mesure d'intégrer toutes ces grandes conclusions de la science du social.

L'approche phénoménologique est essentiellement axiomatique. Elle l'est parce qu'elle est constante habilitation des termes d'un appareillage conceptuel et que cette attitude ne repose pas sur une confrontation de ce qui est répété et de ce que donne à observer le champ empirique. La question se pose pourtant : l'humain est-il si fondamentalement, si absolument intentionnel que ne le soutiennent les protagonistes de l'approche rationalisante ou phénoménologique? La répétition peut convaincre, mais elle ne constitue pas une preuve. L'interconnexion de concepts, si essentielle soit-elle en science, ne présente pas en soi une vérité et n'interdit pas qu'on se soumette à la dialectique de la théorie et des objets de théorie. La démarche que nous adoptons pose la question de l'universalité de l'intention et, plus généralement, des termes de l'approche rationalisante. Son objectif est d'entamer une vérification empirique de ce que prédit cette approche et, en même temps, d'ouvrir un espace plus relationnel à la théorisation. Il nous semble qu'il est devenu nécessaire d'obliger l'approche rationalisante à tirer les conséquences de ses propres postulats, ce qui peut être fait de plusieurs façons. Nous pouvons, par exemple, formuler ainsi ses propositions :

proposition 1: si le discours n'est qu'intention, c'est qu'il n'est que rationnel

proposition 2: s'il y a intention, c'est qu'il n'y a pas de position émorationnelle avant l'échange

proposition 3: s'il y a intention, c'est que l'information ne peut pas naitre de l'échange

proposition 4: si l'échange s'explique en fonction d'une intention, cela signifie que le discours est immuable. 


\section{Méthodologie}

Pour vérifier s'il en est ainsi, nous avons tout simplement choisi d'observer des échanges entre des interlocuteurs pris deux à la fois. Les participants devaient être en mesure de communiquer dans une même langue, en l'occurrence le français, et ils devaient être âgés d'au moins dix-huit ans. Puisque les prétentions de l'approche rationalisante sont universelles, il ne nous est pas apparu utile de prendre en considération des variables d'ordre sociodémographique. Cependant, il nous est apparu opportun de distinguer les dyades selon que les interlocuteurs se connaissaient ou non avant que n'ait lieu l'échange. Cette variable s'est avérée tout à fait inutile pour différencier les résultats; elle n'est donc pas exploitée dans l'exposé qui suit. Chacun des deux échantillons devait être composé de cinq dyades ${ }^{26}$. Les observations ont été faites auprès de la population francophone de l'Ontario.

L'instrument de collecte devait au moins pouvoir enregistrer le discours; mais le non-dit étant souvent révélateur de sens, la saisie a été effectuée sur cassette audio-vidéo $^{27}$. Le thème devait être d'intérêt commun et connu de tous. Il s'agissait de faire ressortir des opinions et des perceptions plutôt que des qualités ou des aptitudes spécifiques relevant d'une spécialisation quelconque; on espérait ainsi ouvrir la voie à la discussion. Le travail visant à étudier le rôle de la relation dans la circulation de l'information entre des personnes, l'artificialité de la rencontre ne posait pas de problème d'ordre théorique. Le thème retenu fut celui du mariage et nous l'avons proposé sous la forme suivante : "Que pensez-vous du mariage ? " Puisqu'il s'agit là d'une composante importante de la culture occidentale, d'un phénomène institutionnalisé, chacun pouvait en parler.

Une fois la saisie des séances terminée, il s'agissait de trouver une méthode qui permette de traiter les données visuellement. Chaque séance fut reproduite par écrit, c'est-à-dire sous forme de verbatim. Il importait,

\footnotetext{
${ }^{26}$ Pour une analyse plus détaillée, on se référera au travail de Mélanie Girard (op. cit.).

${ }^{27} \mathrm{La}$ saisie des séances débuta le 16 novembre 2001 et se termina le 29 mars 2002 . Elle a eu lieu dans le nord de l'Ontario. Le tout s'est déroulé dans une salle aménagée à cet effet. On y trouvait deux chaises placées en face à face que séparait une table. Les sujets prenaient place à la table après avoir eux-mêmes choisi leur siège. Ils remplissaient un bref formulaire de consentement. Une fois le formulaire rempli, on leur expliquait comment se déroulerait la séance. Les caméras en marche et la discussion lancée, l'expérimentatrice se retirait derrière une vitre teintée pour observer l'échange.
} 
tout au long de ce travail, d'être aussi fidèle que possible aux paroles, aux gestes, à la circulation des idées, et d'éviter toute distorsion. Ainsi, dans le but de maintenir la cohérence entre les séquences, une grille a été dévcloppée qui permettait d'appliquer aux énoncés les mêmes règles, une même logique. Au fur et à mesure de la transcription, une série de notes furent prises à des fins de traitement des propos en fonction des récurrences, d'une part, et des exceptions, de l'autre ${ }^{28}$.

Avant de pouvoir entamer l'analyse, il importait de structurer les données recueillies; il fallait donc découper les propos en segments pour soumettre chacun d'eux à l'ensemble des questions analytiques. Nous disposions, pour chacun des deux types de regroupement - selon que les interlocuteurs se connaissaient ou non -, de cinq dyades, pour un total de dix rencontres. Pour chacune d'entre elles, nous avons choisi de retenir cinq séquences à soumettre à l'analyse. Chaque séquence s'articulait à un tour de parole sélectionné aléatoirement à l'aide d'un logiciel (SPSS). Autour de chacun d'eux, nous avons construit une séquence intelligible, c'est-à-dire qui permette de retracer le fil des idées. Une séquence devait être complète en elle-même et, donc, comporter les éléments nécessaires à son intelligibilité ; ainsi, une séquence pouvait inclure des paroles qui précédaient ou qui

${ }^{28}$ Les consignes relatives à la reproduction du discours furent les suivantes : les expressions anglophones furent incorporées au texte. Les guillemets furent réservés aux propos rapportés. Le gras servit à mettre l'accent sur des mots, des groupes de mots ou des expressions. L'italique servit à rendre compte des observations de l'expérimentatrice. Les tirets servirent à illustrer les hésitations, les reprises d'idées antérieures et les coupures dans le dialogue ; ils rendaient donc compte, en même temps, du rythme. Dans la mesure où le tiret servait à indiquer les coupures, les bris dans le discours, il a été utilisé dans les cas où il y avait une interruption ou un changement d'orientation dans les propos, et, bien souvent, cet événement était occasionné soit par une réaction tardive aux propos émis précédemment, soit par l'apparition d'une idée nouvelle. Les points de suspension furent utilisés surtout pour indiquer la réflexion et témoignent donc d'une courte pause. Les majuscules servirent à indiquer l'amorce d'une phrase complète ; elles furent donc utilisées pour rendre compte d'une réponse à une question, à une suggestion ou à une intervention. Puisqu'il s'agissait d'un dialogue où les pauses et les intonations étaient décisives de la ponctuation, nous apposions également une majuscule dans les cas où, bien qu'il ne s'agissait pas toujours d'une phrase complète, nous jugions que le temps écoulé avant l'émission d'une réponse était significatif ; bien souvent, ce laps de temps témoignait d'une réflexion menant à une autre idée et, en cela, il marquait le début d'une nouvelle séquence. 
suivaient le tour de parole sélectionné. Cinquante-cinq séquences furent reconstituées ${ }^{29}$.

\section{Analyse}

Le travail que nous proposons consiste, essentiellement, à vérifier si les propos échangés se veulent le résultat de situations interactionnelles plutôt que le fruit d'une intention quelconque. Il s'agit, concrètement, de révéler en quoi les éléments qui composent l'échange appartiennent à la relation en cours et, en cela, émanent de la dynamique à l'oeuvre, c'est-à-dire d'une relation que génèrent, qu'entretiennent et que concourent à transformer les interlocuteurs. Si nous tenons à établir une telle distinction, c'est que, à l'opposé, inspirés d'une vision plus rationalisante, certains prétendent que l'échange dépend nécessairement d'une intention.

Alors qu'il aurait certes été possible, dans la démarche, de se limiter à cibler, par-delà l'intention, les lieux d'intervention des composantes d'un modèle relationnel, nous avons plutôt choisi de faire la démonstration du contraire, c'est-à-dire de confronter la logique rationalisante aux conséquences de ses propres postulats en appliquant la théorie aux données recueillies.

Nous aurions pu effectuer cette confrontation en prenant chacune des séquences de l'échantillon et en l'obligeant à se positionner par rapport aux quatre propositions que nous avons déjà formulées. Cette manière de faire, toutefois, bien que susceptible d'avoir un fort effet de confirmation à force d'accumulation des résultats, aurait été infiniment redondante puisque, dès

\footnotetext{
${ }^{29}$ Une fois que neuf des dix rencontres aient été saisies, dont cinq regroupant des gens qui se connaissaient, nous avons réuni deux participants pour ce qui devait constituer la dernière rencontre. Alors qu'il devait s'agir d'une rencontre entre étrangers, nous avons constaté que les personnes se connaissaient, qu'elles fréquentaient le même cercle d'amis ; nous avons tout de même saisi l'échange. Il fut décidé que, pour chacun des échanges, cinq séquences seraient retenues ; le nombre d'échanges s'élevant maintenant à onze, nous disposerions, au total, de cinquante-cinq séquences sélectionnées de façon aléatoire. Dans chacune des transcriptions de chacune des rencontres, fut numéroté chaque tour de parole. Aussi, puisque nous voulions vérifier s'il existait une différence dans la façon d'échanger selon que les agents se connaissaient ou non au préalable, il importait de codifier les échanges en fonction de cette variable. Dans les cas où les gens se connaissaient avant la rencontre, l'échange portait la cote " $\mathrm{C}$ ", suivie d'un trait d'union et d'un numéro, selon l'ordre dans lequel elle avait été filmée; dans les cas où les agents communicants n'avaient pas fait connaissance au préalable, l'échange portait la cote "NC », suivie, ici encore, d'un numéro se rapportant à l'ordre de la saisie des rencontres.
} 
la description du deuxième cas, elle n'eût constitué qu'une répétition d'un travail déjà fait. De la même manière, les propositions de recherche sont si intimement liées les unes aux autres que, en montrant comment les données se rattachent à l'une d'elles, on révèle implicitement comment elles sont associées aux trois autres. Par exemple, si l'on illustre comment les propos échangés ne correspondent pas à une logique de l'intention et donc ne sont pas strictement rationnels (proposition 1), on indique par le fait même leur part d'émorationalité (proposition 2), la mesure d'après laquelle ils relèvent de l'échange, ou la capacité de cet échange à se générer lui-même (proposition 3) et, par conséquent, on montre comment l'échange peut se transformer lui-même (proposition 4$)^{30}$.

Nous avons plutôt choisi d'aborder l'échantillon à partir de la première proposition (si le discours n'est qu'intention, c'est qu'il n'est que rationnel) et d'en reconstruire toutes les conséquences empiriques. Six catégories ont ainsi pris forme, catégories qui constitueront aussi, éventuellement, des constats.

\subsection{L'agencement des propos comme fonction des réactions}

Dans la mesure où les acteurs se voudraient, dans une perspective rationalisante, guidés par une intention, elle-même fonction d'un intérêt particulier perceptible dans une stratégie quelconque, leurs propos ne sauraient dépendre les uns des autres. Il importe donc de voir dans quelle mesure les énoncés s'entreproduisent ou non.

Nous avons noté que, dans l'ensemble des cas retenus, les propos émanent effectivement d'un effet de réaction entre les interlocuteurs. Les propos d'un agent « $\mathrm{A}$ » ou, du moins, leur insertion dans l'échange à un moment donné, ne trouvent leur raison d'être que dans et à travers les propos précédents, nécessairement émis par un agent « $\mathrm{B}$ », et ce, dans tous les cas observés. Plus précisément, toutes les séquences sélectionnées placent l'observateur devant des situations fondamentalement interactionnelles dans la mesure où l'interaction - plutôt qu'une intention quelconque - se veut décisive du déroulement de l'échange. L'intention n'y serait pour rien. Un agent « $\mathrm{B}$ » n'émet peut-être ses propos que par réaction à un agent "A », et vice versa. Le discours des agents relève, en partie du moins, de positions qui précèdent l'échange en ce qu'elles étaient probablement présentes avant qu'ils ne se rencontrent et, en cela, il pourrait relever d'une

\footnotetext{
${ }^{30}$ Ce sont les deux approches auxquelles nous avons recouru dans les toutes premières analyses, avant que l'instrument analytique ne soit arrêté.
} 
intention quelconque. Il importe toutefois de souligner que l'échange, beaucoup plus qu'une probable intention individuelle, justifie la circulation des informations.

Illustration (séance C-02, tours de paroles 3 à 15)

A : Bien euh, en parlant de mariage, moi puis $\mathrm{X}$, on en parlait hier-

B : Aahhh! Did you really ??

A : An-han! -

$\mathrm{B}:$ Oh! Elle s'avance, se met davantage à l'écoute.

A : Puis on a décidé que -

$\mathrm{B}:$ - de quelle façon? Avec un petit rire...

A : - bien on a reparlé de qui on veut dans notre mariage -

$\mathrm{B}:$ - ah oui?

A : — parce que tu sais d'habitude là t'as comme trois — uhm, c'est comme si tu as trois filles d'honneur, tu vas avoir trois hommes -

B : - ah oua - oui, oua-ouj -

A : — c'est — ça devrait tout matcher — bien, nous autres, on va avoir trois femmes puis cinq hommes... parce que - bien parce que, moi, je veux avoir mes amies dans le mariage puis, lui, il veut - lui, il veut ses amis, so - puis son frère, puis c'est puis c'est tous des gars, so -

B : - oui-

A : - il y a une fille... comme on est en train de piger pour avoir... bien autant de filles qu'on veut.

Dans cet exemple, il suffit de s'attarder aux deux premiers tours de parole pour constater l'impossibilité de faire abstraction d'un effet de réaction dès lors qu'on veut rendre compte du propos. En effet, lorsque «A » affirme que « [...] en parlant de mariage, moi puis $\mathrm{X}$, on en parlait hier ", c'est une « B » agitée qui s'exclame tout en la questionnant: "Aahhh! Did you really ? »Ce dernier énoncé ne saurait s'expliquer sans le précédent, sans la mise en contexte que fournit l'énoncé initial, émis par " $A$ ». Le concept de réaction, ici, révèle tout son sens, toute sa force, en ce que le fait est perceptible non seulement dans le discours lui-même, mais aussi dans le ton que prend l'interlocutrice, c'est-à-dire dans le fait que « $B$ » s'émeut visiblement devant la révélation de « $A »$.

\subsection{Les réactions comme partiellement émotives}

Dans le but de déterminer la place réelle de la rationalité au sein de l'échange et, par conséquent, l'utilité du concept d'intention, on peut vérifier si les réactions inhérentes aux interactions portent en elles une part d'émotion. L'argument, ici, est le suivant : si l'échange s'explique en fonction des réactions, c'est qu'il ne peut avoir pour cause la seule raison humaine, la réaction, dans les rapports entre les personnes, témoignant 
normalement d'une part d'émotion. Si, donc, les réactions observées portent effectivement en elles quelque émotion, c'est que l'échange ne peut s'expliquer de façon purement rationnelle, c'est qu'il s'explique plutôt de façon émorationnelle et que l'intention est peu utile pour rendre compte de la complexité des interactions et, par extension, des relations humaines.

Sur les cinquante-cinq séquences retenues, quarante-cinq comportent des signes évidents d'émotion ; autrement dit, il est possible de repérer en elles - dans les énoncés eux-mêmes, dans les gestes, dans les intonations un espace - nécessairement non uniforme bien que cela n'ait d'importance réelle que dans la mesure où l'on voudrait faire de l'échange un projet strictement émotif, ce qui n'est pas le cas ici - qui relève du domaine de l'émotivité. Dans les cas où l'émotion n'est pas directement perceptible, elle l'est tout de même de façon indirecte en ce qu'il suffit d'examiner soit les propos qui précèdent, soit ceux qui suivent la séquence sélectionnée pour détecter, encore une fois, des éléments attribuables au caractère émotif de ce qui est communiqué.

Illustration (séance NC-02, tours de paroles 92 à 110)

B : Oui, je pense pas qu'il y a des règles parce que mes — mes parents ont — se sont rencontrés puis ils se sont mariés dans quatre mois -

A : - n - oh my gosh ! -

B : — puis ils sont ensemble encore pour trente ans, so, il n'y a pas de - de fast rule là -

A : - oui - oui-

$\mathrm{B}:$ - que tu peux te diriger vers, mais -

A : - est-ce qu'ils disent que c'est euh, love at first sight ou...?

B : Non, il y a - il y a, ouin — non, je pense pas que - ils ont dit que c'était un vrai struggle euh... euh, l'amour, ça - c'est - mon père, il dit qu'il était un petit peu euh, lonely?

A : Oui.

B : puis euh, il était dans l'armée -

A : ahhhh! — comme si elle venait de constater quelque chose

$B$ : — puis il a rencontré ma mère, puis, là... ma mère était une infirmière -

A : — ahhh! - elle le dit d'un ton qui laisse paraitre sa sensibilité, le fait qu'elle trouve cela bean

B : - puis son — c'était le temps, le — un événement puis tout ça qui a — s'est arrangé tout pour eux autres, puis -

A : - uhm-uhm -

B : - après ça puis... c'était difficile puis... mais je pense qu'il y avait beaucoup de support dans ce temps-là, euh, ils — ils m'ont dit qu'ils — ils se — savaient pas s'ils seraient ensemble encore si c'était dans ce temps ici-

A : - oué -

B : - parce qu'il y a plus de choix maintenant -

A : - uhm-uhm -

B : - il n'y avait pas de choix parce que tu te mariais, tu restais marié, puis tout ça... 
L'interlocuteur « $B$ » partage, avec l'interlocutrice « $A$ », l'histoire amoureuse de ses parents ou, plus précisément, les circonstances entourant leur rencontre initiale et leur union subséquente. Les premiers propos, dans cette séquence, sont émis par «B», qui explique que ses parents, ensemble depuis maintenant trente années, se sont mariés quatre mois après leur première rencontre. Il en déduit qu'il n'existe pas de règle universelle, de formule magique, pour la réussite du mariage. À la toute première affirmation de « $\mathrm{B}$ » où il fait allusion à la brièveté de la période pendant laquelle ses parents se sont courtisés, « $A$ » répond par un « [...] oh my gosh !», c'est-àdire par une exclamation, une expression qui témoigne de son étonnement. Déjà en cela, nous sentons que les propos émanent d'un répertoire au moins aussi émotif que rationnel puisque, si elle s'émeut ainsi, c'est que le phénomène lui apparaît étrange, inhabituel. Dans le même esprit, si le phénomène lui apparaît insolite, c'est qu'il s'insère mal dans son propre cadre de références, dans ses propres coutumes. Si le discours de l'interlocutrice est en partie fonction de ses valeurs, il est également fonction de sa dimension émotive, il fait appel à sa socialisation, à son vécu et, par conséquent, à divers aspects de sa psyché, à sa conscience et à son inconscience, à ce qu'elle affirme logiquement, à ce qu'elle ne peut expliquer rationnellement, à ce qui la touche malgré elle, aux paradoxes qu'elle véhicule, à ce qu'elle comprend, à ce qu'elle ne comprend pas et, donc, à tout son univers émorationnel. Les valeurs transitent par le vécu, elles se matérialisent entre l'émotion et la raison, elles apparaissent dans les réactions des agents communicants. Bien qu'elles soient partagées par nombre de personnes et qu'elles soient, en cela, collectives, elles ne s'activent que parce que des individus les reproduisent et les produisent dans l'action communicationnelle.

Cette illustration est éloquente. Mais la séquence peut contribuer encore davantage à révéler la présence de l'émotion. Dans la question que pose « $A$ » tout de suite après, elle demande à « $B$ » s'il s'est agit, pour ses parents, d'un coup de foudre, de l'amour au premier coup d'œeil. On retrouve bien alors la contribution de l'émotion dans les échanges ; si tel n'était pas le cas, comment pourrait-on expliquer qu'elle fasse intervenir un sentiment plutôt qu'un argument rationnel en vue de donner quelque signification à une situation qu'elle comprend difficilement de par la distance symbolique entre ses valeurs et celles des parents de $\langle\mathrm{B} »-$ dont elle rend compte ? Comment même expliquer qu'elle établisse un lien entre mariage et émotion autrement qu'en tant qu'elle est socialisée, réalité qui rappelle ici que les symboliques peuvent changer d'une société à l'autre, d'une époque à l'autre? 


\subsection{L'impossibilité de réduire l'émotion à une intention}

L'émotion est inhérente aux échanges que nous observons. Mais est-elle, elle-même, révélatrice de l'intention qu'entretiendraient - soit de façon ouverte, soit de façon discrète - des agents animés par une volonté de tirer profit de la situation? Si nous répondons par l'affirmative, l'analyse prendra fin, l'intention dictant l'ensemble des interactions observées et les concepts propres au modèle relationnel se révélant inopérants. Or, ce n'est pas ce qui se donne à l'observation : dans quarante des cinquantecinq séquences, il apparaît douteux, voire improbable que l'émotion soit associée à une intention quelconque. Pour ce qui est des quinze autres séquences, un tel questionnement doit être considéré comme étant sans objet puisque l'émotion n'est pas immédiatement perceptible, figurant en amont ou en aval de la séquence examinée. Nous constatons, dans l'ensemble, que les propos se construisent les uns par rapport aux autres au fur et à mesure que l'échange progresse, qu'ils naissent d'idées que déclenchent, chez un agent « $\mathrm{B} »$, une parole, un geste d'un agent « $\mathrm{A} »-$ ou vice versa. Ce qui relève de l'ordre de l'affectif semble devoir s'expliquer par référence à un cadre socioémotif, socioculturel, sociohistorique. L'intention, encore une fois, ne permet de rendre compte ni des éléments propres à l'échange, ni de l'interaction des agents communicants.

Illustration (séance C-02, tours de paroles 394 à 406)

$\mathrm{B}:[. .$.$] parce que tout le monde veut qu'ils se marient! Ah, seigneur, lui, il veut la$ marier! -

A : — oui, mais, elle, elle veut pas -

B : - bien, c'est —

A : — Hummmmm — son ton est insinuateur, même presque arrogant

B : - bien c'est - c'est pas qu'elle veut pas le marier -

A : — ça a l'air à être la même chose dans toute ta famille ou quoi ? - elle rit en le disant

Pause.

$\mathrm{B}:$ Anh? - A rit - Anh? Quoi ? - son ton n'est pas plaisant, elle ne semble pas comprendre ce que $A$ essaie de lui dire

A : Je vois comme une euh - elle rit plutôt que de terminer sa phrase

$\mathrm{B}$ : Il n'est pas arrivé la même affaire avec moi! — son ton est defensif

A : Non ? X...? -

$\mathrm{B}$ : Oh well, pfff — avec un signe de la main comme si elle s'en fichait — whatever! Eum, elle — A rit — non elle - soupir — c'est parce qu'elle aime ça être libre -

A : - ouin -

$\mathrm{B}:$ - elle, elle acte son âge.

L'interlocutrice « B » explique à « A », en parlant de sa sœur et de son copain, que leur entourage souhaite qu'ils se marient, que le copain, lui, 
veut épouser sa sœur. «A » l'interrompt aussitôt : « oui, mais, elle, elle veut pas ». « $\mathrm{B}$ » s'apprête à expliquer quelque chose, mais elle en est aussitôt empêchée par « $A$ » qui se contente de faire "Hummmmm », pensive, insinuatrice, presque arrogante. « $B$ » reprend à nouveau, mais sans succès puisque « $A$ » lui coupe la parole pour une troisième fois et pose en riant une question qui sera reçue un peu comme une accusation. À cette question « ça a l'air à être la même chose dans toute ta famille ou quoi ? » elle-même suivie d'une pause, « B » se montrera hésitante, voire déconcertée et exigera des renseignements supplémentaires à l'appui de ce qu'on avance à son égard. " A " ne terminera toujours pas son explication; elle se mettra à rire. « $\mathrm{B}$ » lancera, à la défensive, que la même chose ne s'est pas produite avec elle, ce sur quoi « $A$ » lui rappellera, en évoquant un nom, un événement passé, évocation à laquelle « $B$ » réagira comme par déni pour, enfin, revenir au thème initial, soit son interprétation des raisons pour lesquelles sa sœur ne désire pas le mariage.

Dès le premier tour de parole, on entrevoit de l'émotion dans l'usage du verbe vouloir qui traduit ici des aspirations qu'on ne saurait réduire à une pure déduction logique. Mais ces désirs ne sont pas ceux de l'interlocutrice et l'on ne peut directement apercevoir l'effet qu'ils ont sur elle. L'émotion apparaît toutefois chez les agents communicants dès que « $A$ » fait emphatiquement «Hummmmm » de façon soupçonneuse. On la perçoit davantage lorsque, en guise de réponse, « $\mathrm{B}$ » s'apprête à livrer une explication qu'entrecoupe l'agent « $A$ » en disant « ça a l'air à être la même chose dans toute ta famille ou quoi ? », assertion qui produit un effet que « $A$ » camoufle par le rire.

Les émotions transparaissent dans le jeu des répliques, dans les gestes, dans les attitudes, dans les mots, dans les sons. On peut se demander si elles relèvent de l'intention. S'il en est ainsi, c'est-à-dire que si les émotions découlent d'une intention spécifique, il faut identifier cet objectif, ce motif que masque un échange en apparence spontané. Et quelle serait l'intention de « $A$ »? De narguer « $B$ » en dépeignant sa situation familiale comme étant problématique? Mais alors pourquoi s'arrêtera-t-elle au beau milieu de sa phrase un peu plus loin ? Dans quel but, puisque tout projet rationnel porte nécessairement en lui une visée spécifique? Serait-ce qu'elle désire la ridiculiser? lui faire du tort? la faire rire? lui faire prendre conscience d'un contexte familial dysfonctionnel ? Peut-être! Mais on peut en douter. La question ironique de « $A$ » sera suivie d'une pause, pause après laquelle « $\mathrm{B}$ » ripostera, d'un ton qui laisse entrevoir un certain degré d'indignation, en exigeant que « $\mathrm{A}$ » répète ce qu'elle vient de dire. Elle le fera à trois reprises, maintenant toujours un air de contrariété, 
d'agacement. " $A$ » tentera de fournir une explication - «Je vois comme une euh »- le rire, encore une fois, l'animera et elle évitera ainsi la discussion. Quelle serait la fonction du rire dans ce cas-ci ? Si l'intention, au début, consistait à confronter « $\mathrm{B}$ » à sa situation familiale, faut-il en déduire qu'elle abandonne, ici, sa poussée initiale ? Serait-ce, encore, qu'elle choisit de terminer sa phrase de façon à laisser « $B$ » tirer ses propres conclusions de l'idée qui a été entamée ? Et alors serait-ce que l'intention initiale s'est modifiée ? Et si l'intention initiale s'est transformée, a-t-on toujours affaire à une intention ou doit-on plutôt faire appel à des concepts additionnels, plus précis, plus riches pour décrire de telles évolutions, plus communicationnelles que subjectives ? Si l'on adoptait la position de l'agent « $\mathrm{B}$ » et que l'on tentait d'expliquer l'échange en fonction d'une intention de sa part plutôt que de celle de " $A$ », comment justifierait-on un énoncé initial indiquant que « tout le monde veut qu'ils se marient! Ah seigneur, lui, il veut la marier »? Par recours à l'intention? Et quelle serait cette intention? De convaincre « $A$ » de l'authenticité du fait ? Et si elle désire ainsi persuader l'autre, ce serait dans quelle perspective? La sienne? Celle du copain? Autrement dit, est-ce de la véracité de son affirmation qu'elle veut convaincre - « tout le monde veut qu'ils se marient» - ou de celle du copain - « ah seigneur, lui, il veut la marier »- ? Et pourquoi ? Dans quel but? Admettons pour l'instant que « $\mathrm{B}$ » communique dans l'optique de convaincre que tout le monde, y compris le copain, désire le mariage. Rangeons-nous, de façon à simplifier les choses, du côté du copain. L'intention de « $\mathrm{B}$ » serait donc de convaincre « $A$ » que le copain désire vraiment épouser la sœur. Alors, il serait possible, en principe, de détecter, parmi les émotions dont on observe plus loin la manifestation, des indices de cette intention. À travers eux, nous devrions trouver, dans l'ensemble du propos, une certaine convergence révélatrice d'une stratégie. Mais qu'apercevons-nous véritablement? La cohérence n'y est pas. Le commentaire suivant de « $\mathrm{B}$ » - «bien, c'est» - semble beaucoup plus une allusion à l'interruption de « $A$ »- « oui, mais, elle, elle veut pas »- qu'il ne fait suite à une idée précédente; de même, deux tours de parole plus loin, « $\mathrm{B}$ » semble toujours dans une logique interactionnelle lorsque, suite à l'interjection de «A », elle reprend son explication en l'élaborant. Plus loin, encore, elle réagit au commentaire ironique de « $A$ ». Où serait alors l'intention ? Et que dire de l'apparente indignation dans le propos qui n'entretiendrait pas de lien logique avec cette intention? Est-ce qu'une nouvelle intention est née de l'échange et peut naître de lui ? Comment alors penser l'intention? Comme muable, instantanée, éphémère ou inconséquente? Pourquoi ne 
pas parler, plutôt, d'un effet d'adaptation à la situation, d'animation par des symboles communs, familiers, issus d'un répertoire historique, social, à la fois émotif - dans la mesure où l'on y trouve l'adhésion à des valeurs - et rationnel - puisqu'un argument peut être conçu et qu'une position peut prendre forme? Si, finalement, à l'opposé, nous retenons l'opinion selon laquelle « $B$ » désire persuader du fait que l'entourage de sa sœur souhaite qu'elle et son copain se marient, alors nous avançons dans la même impasse : vouloir rendre compte, à partir de l'appareillage conceptuel corrélatif de la notion d'intention, du fait que « $\mathrm{B}$ » veuille persuader « $\mathrm{A}$ » de l'authenticité du désir d'une foule de gens de voir le couple consacrer une union par le mariage n'identifie toujours pas l'intérêt de « $B$ », c'est-à-dire que cela ne montre pas en quoi elle en tire quelque profit.

Il parait peu probable qu'une logique rationalisante puisse expliquer que le malheur de l'un - celui qui veut épouser celle qui ne le veut pas provoque l'amusement de l'autre - «A $\mathrm{A}$ dans ce cas-ci - alors que cet amusement n'est associable qu'aux symboles qui rappellent la situation à la personne qui en rit. Ne serait-il pas ici plus utile de faire état de l'incidence de symboliques communes chez des agents communicants à la fois semblables et différents? Ne percevons-nous pas encore cette identification à des références communes dans la défense de « $\mathrm{B}$ » qui, sans même en avoir été accusée clairement, soutient que la même chose ne lui est pas arrivée, ramenant ainsi un commentaire d'ordre général « la même chose dans toute ta famille »-au particulier, à elle-même ? Comment prétendre pouvoir ramener des expressions aussi flagrantes d'appartenance culturelle à l'intention, à l'intérêt, à la simple rationalité de l'humain? Comment justifier qu'une modélisation omette une composante aussi importante, aussi intégralement humaine que la sensibilité, que l'émotion?

\subsection{L'émotion comme participant de la socialité}

Après avoir déterminé que, dans l'échange, ce sont les réactions - plutôt que l'intention des agents communicants - qui modulent la circulation de l'information et que ces mêmes réactions sont issues d'un répertoire partiellement émotif, il est sans doute nécessaire de s'interroger sur les éléments qui interviennent sur les émotions et, par conséquent, à partir desquels on parvient à les reconnaitre. L'observation ayant permis d'apercevoir leur apport dans les échanges, elle a, par le fait même, accentué la mise en doute d'une communication fondamentalement 
déterminée par l'intention des acteurs sociaux. Au point de départ, il avait été entendu que si nous devions découvrir que l'émotion est déterminante, que la rationalité n'est pas suffisante, le concept d'intention comme catégorie essentielle de l'explication de l'échange entre les personnes devrait, pour le moins, être remis en question. Il faudrait alors, selon nous, déplacer l'appareil analytique vers un modèle plus relationalisant qu'individualisant. La question qui se pose consiste à savoir si l'émotion qui se dégage dans chacun des exemples porte en elle une part de socialité, c'est-à-dire une partie composée d'éléments qui seraient familiers aux deux agents impliqués dans l'échange de par leur appartenance à un contexte social commun. Au plan opérationnel, il s'agit de relever, dans les interactions, les références à des symboliques sociales.

\section{Illustration (séance C-03, tours de paroles 168 à 178)}

A : Non mais j'aimerais bien être demoiselle d'honneur ; moi, j’ai raté tous les mariages de toute ma famille!

$\mathrm{B}:$ Tu as jamais été ?

A : Non -

B : - ooohh! -

A : - tout le temps qu'il y a... - chaque fois qu'il y avait un mariage, ça tombait au

— durant l'année scolaire et, moi, j'étais en France -

B : - ooohh! -

A : - et, tu vois... et tout le monde partait au Sénégal et, moi, je pleurais... Ellle commence à rire et $B$ enchaîne; $B$ rit à gorge déployée pendant que $A$ poursuit : je te dis, je te dis, j’ai pleuré combien de fois; on m'appelle : « oh regarde, elle s'est — elle s'est mariće »; je regarde la caméra et je veux pleurer -

B : - aaaaahhh! -

A : — je regarde la cassette -

B : - uhm-uhm -

A : - je suis dégoûtée, j'ai - j’ai toujours raté les mariages...

« $A$ » amorce la séquence en signalant qu'elle aimerait être demoiselle d'honneur ; elle ajoute par la suite qu'elle a raté tous les mariages de sa famille. On sent, de par l'intonation, qu'elle en est navrée ; on sent également, chez «B », une certaine surprise, un certain étonnement lorsqu'elle confirme les propos de « $\mathrm{A} »-$ « Tu as jamais été ? -, étonnement qui se transforme vite en désolation lorsque « $A$ » explique que, si elle a raté tous ces mariages, c'est qu'ils avaient lieu durant l'année scolaire, au Sénégal, alors qu'elle étudiait en France. Elle dit avoir pleuré pendant que tout le monde partait pour le Sénégal. En le disant, elle se met à rire et « $\mathrm{B}$ » ne tarde pas à l'accompagner. " $\mathrm{A}$ » souligne avoir pleuré de nombreuses fois pour ce motif, d'autant plus qu'on lui expédiait chaque fois une cassette vidéo lui donnant à voir ce qu'elle avait manqué. Elle termine en parlant de son envie de pleurer lors des visionnements et 
se dit " dégoûtée » de n'avoir pu assister aux mariages. Les derniers énoncés sont entrecoupés des exclamations de «B 》. Les émotions qui transparaissent dans le dialogue s'insèrent, à notre avis, dans un cadre essentiellement historique. En cela, elles se veulent fondamentalement sociales ou, pour dire autrement, porteuses de socialité. Prenons, à titre d'exemple, le premier énoncé, où « $A$ » affirme qu'elle aimerait être demoiselle d'honneur et signale qu'elle a raté tous les mariages qui ont eu lieu dans sa famille. Que « $A$ » désire être demoiselle d'honneur n'a de sens que dans la mesure où « $B$ » sait ce qu'est une demoiselle d'honneur et en vit la symbolique de façon affective. Autrement dit, si l'information émise par « $A$ » peut être reçue dans sa signification profonde, c'est que les agents partagent des représentations qui rendent intelligibles à la fois les propos soulevés et leur agencement. Si, donc, «B » s'exclame devant la déclaration de « $\mathrm{A}$ » qui, elle, dit n'avoir jamais eu la chance d'être demoiselle d'honneur et déplore avoir raté tous les mariages de sa famille, c'est, d'abord, qu'elle comprend l'importance pour «A »- en tant que jeune femme, en tant que Sénégalaise... - de faire ainsi partie des noces ; c'est, ensuite, qu'elle reconnaît, du même coup, son désir d'assister à de telles cérémonies, le mariage étant ici synonyme de célébration, de rassemblement, de rites spécifiques et connotés. De la même façon, si, lors de sa présentation des raisons pour lesquelles elle a été empêchée de participer aux cérémonies en question, " $A$ » se contente de dire que " ça tombait [...] durant l'année scolaire et, moi, j'étais en France», c'est qu'elle n'a pas à expliquer en quoi une année scolaire consiste, pas plus qu'à exposer les raisons pour lesquelles elle ne s'autorisait pas une pause dans ses études, pas plus même, qu'à rendre compte de la distance entre la France et le Sénégal, ces informations étant toutes implicites de par la relation qui existe entre les interlocutrices, d'une part, et, d'autre part, de par la socialité qu'elles portent en elles, socialité qui déborde leur relation immédiate pour les inscrire dans un contexte plus global où les symboles prennent un sens spécifique. Il ne peut s'agir ici d'un simple effet du hasard si « $\mathrm{B}$ » se met à rire en même temps que « $\mathrm{A}$ » quand cette dernière raconte avoir pleuré le départ des membres de sa famille. Si le rire de « $A$ » entraine celui de « $B$ », c'est qu'il renvoie à des référents communs ou que « $B$ » exprime son empathie envers « $A »$. Dans l'un ou l'autre de ces cas, le caractère social des agents rend possible les conduites. De plus, si les larmes de " $A$ » amusent les deux interlocutrices, c'est que leur rôle est en l'occurrence révélateur d'éléments qui débordent le cadre limité de l'histoire qui est racontée, voire de leur interaction immédiate. 


\subsection{L'émotion comme participant d'une historicité spécifique}

L'analyse précédente avait trait aux lieux d'intervention de la socialité dans les émotions qui habitent l'individu et donnent un sens à l'échange. Cette analyse en appelle une autre qui devrait révéler que, empiriquement, l'échange porte en lui une historicité puisque les catégories analytiques présentées plus haut sont interreliées, l'émorationalité étant attachée et à la socialité et à l'historicité. Pour des fins d'intelligibilité, il nous apparaît nécessaire de distinguer entre historicité spécifique et historicité non spécifique. La première a trait à la relation dans son immédiateté, au rapport qu'entretiennent entre eux dans sa particularité les agents communicants ; elle concerne une histoire spécifique qui est en train de se faire. La seconde a pour objet les catégories médiates qui rendent possible l'échange; elle apparaît dans les informations que portent en eux les agents communicants du fait qu'ils s'inscrivent dans une histoire qui transcende des événements particuliers ; dans une large mesure, c'est grâce à ce registre que l'intercompréhension est possible.

Nous avons perçu dans cinquante et une des cinquante-cinq séquences l'intervention de cette historicité spécifique. Dans chacun des cas, des éléments propres à la relation elle-même contribuent à définir et à modeler l'échange ainsi qu'à faire en sorte que la communication se produise dans son originalité.

Illustration (séance C-01, tours de paroles 134 à 150)

A : - comme je l'ai dit, ça me dérange pas si -

B : - ouais -

A : — mon copain vient me demander le mariage -

B : - oui, oui, ça te dérange pas -

A : — mais si je vois aussi, en même temps, comme la famille, ça serait bien pour eux, parce que, pour elle plutôt eum -

$\mathrm{B}:$ - oui -

A : - parce qu'elle a l'air d'avoir — les paroles suivantes sont prononcées plus bas, presque en un murmure, comme détaché - quand même une assez grande influence sur le mariage -

B : —oué-

A : - mais je pense que je vais le faire -

$\mathrm{B}:$ - tu le ferais --

A : - je le ferais, pas parce que...

B : ... quelqu'un d'autre donne le droit de le faire -

A : —oui, mais -

$\mathrm{B}:$ - tu le ferais pour ton compte, pour tes propres intérêts, pour tes euh — il poursuit mais $A$ a déjà commencé à parler et les deux demiers mots de $B$ sont donc inaudibles

A : — ça me dérange pas de le faire, mais juste le fait que... mes parents ou ma famille -

B : - ouais, ouais -

A : — veut que je le fasse... 
On assiste à une discussion où « $\mathrm{A}$ » exprime son indifférence à l'égard d'une demande en mariage hypothétique de la part de son copain, après quoi elle ajoute qu'elle voit également en quoi une telle situation serait acceptable pour " la famille » parce que, d'après elle, cette famille semblerait exercer une « influence sur le mariage ». Ces dernières paroles, elle les prononce plus bas, presque en un murmure. Devant cette assertion, « $\mathrm{B}$ » répond simplement un " oué », comme s'il avait compris, instantanément, l'importance de cette influence dont elle parle. " $A$ » poursuit en expliquant qu'elle espère se marier. Une intervention de « $\mathrm{B} »$, où l'on sent qu'il veut deviner pourquoi elle pense ainsi, la fait dévier. Elle tente alors d'expliquer pourquoi elle ne voudrait pas se marier, mais, pour une raison quelconque, elle s'interrompt, laissant son opinion en suspens et, par conséquent, ouverte à l'interprétation. « B » tente, ici encore, de deviner ce que dit « $A$ » et propose que « quelqu'un d'autre donne droit de le faire ", ce à quoi elle répond par un " oui, mais » qui est vite interrompu par « $\mathrm{B}$ » qui ajoute : «tu le ferais pour ton compte, pour tes propres intérêts, pour tes euh ». Avant qu'il n'ait pu achever ses paroles, « $A$ » reprend : « ça me dérange pas de le faire, mais juste le fait que... mes parents ou ma famille »; «B » intervient : « ouais, ouais »; « $A$ » conclut : "veut que je le fasse... ».

La notion d'historicité spécifique permet de constater que, si les agents peuvent s'entendre en faisant l'économie de certains éclaircissements que l'on jugerait autrement nécessaires à la compréhension, c'est qu'il existe des éléments qui leur sont communs : au niveau, par exemple, de la langue, des symboliques sociales, du vécu. Autrement dit, c'est parce que les interlocuteurs vivent une relation spécifique que des agents parviennent à entretenir un dialogue dont le sens véritable ne peut être capté par des parties étrangères à l'échange. S'il en est ainsi, c'est que la relation qu'ils entretiennent dépend directement des éléments qui leur appartiennent en propre. Prenons, par exemple, la section de la séquence où « $A$ » affirme que la famille semble influente. Après avoir affirmé qu'une demande en mariage de son copain ne lui déplairait pas, elle ajoute qu'elle reconnait en même temps en quoi cela serait bien vu de sa famille. Or, avant qu'elle n'ait pu élaborer, « B » fait « oui ». Cette affirmation n'est pas retenue sur le champ par « $A$ » qui, dans son élan, poursuit son idée, bien que de façon moins vigoureuse, baissant le ton, ce qui témoigne peut-être d'une incertitude. Pour dire autrement, lorsque « $\mathrm{B}$ » acquiesce, il la surprend probablement. « $A$ » semble alors devenir moins certaine de son affirmation, d'autant plus que « $\mathrm{B}$ » parait maintenant en savoir plus long 
qu'elle sur un sujet qu'elle a elle-même amorcé, et ce, dans la mesure où il donne l'impression de comprendre quelque chose qu'elle n'a pas encore formulé, quelque chose qu'elle ne se sent, tout à coup, pas entièrement en mesure d'expliciter. Puisqu'elle doit, néanmoins, achever ses pensées, elle poursuit; mais elle le fait, comme cela a été mentionné plus haut, de façon atténuée, plus à titre d'hypothèse que d'affirmation. Une seconde confirmation de la part de $" \mathrm{~B}$ » lui donne un coup de pouce utile et lui permet de se reprendre en main, lui donne l'impression d'être comprise, que ses idées sont acceptables, voire partagées et, ainsi, lui fournit les conditions propices à la poursuite de son idée. Par cette reprise, " $A$ " indique qu'elle est ouverte à l'idée du mariage. Cependant, elle est interrompue, ici encore, par « $\mathrm{B} »$ qui, dans un effort d'interprétation, reprend ses paroles, à lui, pour être arrêté à mi-chemin par « $\mathrm{A}$ » qui, encore une fois, dévie de sa trajectoire discursive. Elle relativise alors, évoquant des raisons qui la pousseraient à ne pas opter pour le mariage.

Ce dialogue, qui, de prime abord, peut paraitre anodin, est, au fond, riche de sens. « $A$ » n'a pas le temps de terminer sa première phrase que « $\mathrm{B}$ » lui coupe la parole, comme si tout ce qu'elle allait dire par la suite était déjà compris. Comment peut-il deviner ce que « $A$ » veut dire alors qu'elle ne l'a pas encore exprimé ? Comment sait-il ce qu'elle veut dire quand elle indique qu'une demande en mariage serait perçue favorablement par sa famille alors qu'elle n'a pas eu la possibilité d'établir pourquoi ? L'interruption est vite camouflée par l'élan de «A $A$ qui ne semble pas en tenir compte et qui voudra préciser en quoi le geste en question plairait à sa famille. Cependant, elle a tout juste le temps d'affirmer " parce qu'elle a l'air d'avoir quand même une assez grande influence sur le mariage » que « $\mathrm{B}$ » intervient encore pour faire entendre un « oué », symbolisant, ici encore, l'assimilation d'une idée dont le développement est absent, sinon implicite. Cette seconde interruption en ce qui a trait, particulièrement, au thème de la famille - nous ramène à la notion de complicité dans la mesure où, encore une fois, la compréhension semble provenir d'une dynamique pré-échange qui s'actualise dans la communication en cours. $\mathrm{Si}$ « $\mathrm{B}$ » est en mesure de prendre position sur des propos inachevés sans rebuter «A», c'est nécessairement qu'il devine ce que « $A$ » veut dire sans qu'elle ait à l'expliciter. Si, encore, il peut deviner les sentiments, le propos de son interlocutrice, c'est qu'il a accès à un répertoire particulier, qu'il dispose d'informations antérieures à l'échange lui-même, mais arrimées à la spécificité de leur relation. 


\subsection{L'émotion comme participant d'une historicité non spécifique}

S’il est possible d'isoler les éléments qui relèvent de l'historicité spécifique des agents, c'est qu'il existe, au-delà d'elle, une historicité plus globale ou générale qui la rend possible. Il ne peut y avoir d'histoires particulières que dans la mesure où elles trouvent des conditions de possibilité générales. Cette historicité non spécifique fournit des structures dans lesquelles s'inscrivent des relations. Sans histoire globale dans laquelle se produisent des référents culturels, il ne peut y avoir d'échanges particuliers, bien que ces échanges contribuent à la production et à la reproduction de ces référents. L'immédiateté de l'échange s'articule nécessairement à sa médiateté. Même si la relation revêt un caractère distinct, elle témoigne du même coup de l'appartenance à une communauté, à une culture, à un contexte. Le recours des agents communicants à des symboliques communes qui ne sont pas réductibles à leur propre échange oblige la modélisation à tenir compte du principe d'une historicité non spécifique.

Illustration (séance C-05, tours de paroles 211 à 237)

B : il y a une affaire aussi : c'est que, en étant séparés, c'est toujours embêtant les mariages... comme euh, là tu vas avoir euh, mon ex puis moi, puis, tu sais, ça complique les mariages, ça, là, là...

A : Ah, okay...

B : W souvent me disait : « je vais aller me marier en dehors, c'est trop compliqué !» A : Oué ? Ah oué...

Rires

A : Ah, pour le monde que tu vas inviter puis...?

B : Oué...

A : Ah ! okay... ah ! j'avais pas pensé à ça...

B : Oué... cet aspect là, euh... ah malgré que quand tu es séparé ça fait bien des années... mais il reste que c'est quand même euh « awkward », tu sais ?

A : Oué... acquiescement par signe de la tête.

B : Tu sais, on pourrait dire... mais, ça m'inquiétait beaucoup cet aspect-là, puis l' voilà deux ans, on est allés au mariage du fils à X... bon bien, moi puis $\mathrm{X}$, ça fait vingt ans qu'on est ensemble ; puis $\mathrm{Y}$, bien elle a... elle a... bien elle a marié $\mathrm{Z} . .$. Là, je me demandais : «comment ça va aller les photos puis tout ça, là, tu sais ? » Puis, finalement, ça... ça a bien été, mais parce qu'on avait les mêmes idées sur euh, sur ces affaires là, là, tu sais ?

A : Ah, okay.

B : ... comme $Y$, elle, voulait pas se faire poser avec $X$ toute seule, puis les enfants. Elle rigole un peu en le disant. Elle disait: «Non, non, moi, je me fais poser avec mon conjoint, ça fait assez longtemps qu'on est ensemble !» Mais, là, si, elle, elle avait tenu à se faire poser avec les enfants, juste eux autres, moi, je le sais pas si je me serais sentie euh... je me serais sentie à part, peut-être...

A : Ah! okay.

B : ... je sais pas... C'est ça que je hais, c'est les - 


\section{MÉLANIE GIRARD ... / LINTENTION EST-ELLE SI UNIVERSELLE ...?}

A : Oui, bien c'est ça, c'est d'autres familles qui se sont reformées là, hein...

B : ... ouin...

A : ... c'est ça, vous avez eu des enfants ensemble, là, puis...

B : Oui, bien, là, on en a passé une, noce, ça a bien été, là... mais c'est pas grave, ça, se casser la tête, comme un an d'avance, savoir comment ça irait là... c'est...

A : Oué.

\section{Longue pause.}

B : ... mais j'ai trouvé ça beau...

A: Oué?

B : parce que, moi, $j$ ’aime pas bien ça aller dans les noces, là, mais j’ai trouvé ça beau...

A : C'était $V$, ça, hein?

B : Oui. C'était dehors...

A : ah oui?

B : oué... c'était vraiment beau, c'était simple...

A : oué

Dans ce dialogue, il est question de familles reconstituées, phénomène qui ne peut être abordé avec autant de simplicité que dans un contexte historique où l'événement n'est pas exceptionnel et où il n'est pas stigmatisant. « $B$ » rapporte que, en raison des séparations, les cérémonies de mariage deviennent embêtantes, compliquées. Trouvant le mariage traditionnel « trop compliqué ", certaines personnes - dont une en particulier qui lui sert d'exemple mais avec qui le lien d'affiliation n'est pas spécifié - préfèrent se marier loin des leurs. « $A$ », incertain du sens des affirmations, demande des éclaircissements - «Ah, pour le monde que tu vas inviter puis... ? »- et, après une confirmation de « $B$ », avoue n'avoir jamais songé au problème. « $\mathrm{B}$ » poursuit en expliquant que, même si plusieurs années se sont écoulées depuis la séparation, le rassemblement que requiert la cérémonie du mariage demeure incommode, gênant. Elle raconte ensuite en quoi cet aspect l'inquiétait jusqu'au jour où, il y a de cela quelques années, elle a eu à affronter une telle situation lors du mariage du fils de son conjoint. Elle s'étend quelque peu sur les détails de la journée, sur les pensées qui lui ont traversé l'esprit, sur les sentiments qu'elle a vécus, pour en arriver à un état d'incertitude - «... je sais pas... »-qui débouche sur une prise de position - «C'est ça que je hais, c'est les ». Sa phrase est interrompue par « $A »-«$ Ouin, bien c'est ça, c'est d'autres familles qui se sont reformées là, hein... ». « $B$ » reprend, puis signale que le tout s'est bien déroulé en dépit de ses inquiétudes. Elle reprend une idée laissée en suspens - «[...] mais c'est pas grave, ça, se casser la tête comme un an d'avance, savoir comment ça irait là... c'est... »- dont le sens est évident pour « $A$ » qui, lui, se contente d'approuver. La discussion est suivie d'une longue pause à laquelle « $\mathrm{B} »$ met fin en revenant sur la cérémonie de mariage du 
fils de son conjoint, sur le fait que, malgré son appréhension, l'événement lui a plu. « $A$ » s'assure qu'elle parle toujours de la même personne, ce que « B » confirme pour rappeler, ensuite, que la célébration a eu lieu à l'extérieur, qu'elle était simple et adaptée.

Cela étant dit, nous notons que, dans le cas à l'étude, où l'existence d'une relation pré-échange est évidente à la.lumière des allusions à des connaissances et à des lieux communs, les agents bénéficient non seulement d'une connaissance générale du rapport au social de l'autre, mais aussi, et plus précisément, d'une connaissance spécifique, acquise à travers la relation ; ainsi, en plus des catégories médiates qui sont mises à leur disposition, ils jouiraient de l'accès à des informations ou à des catégories immédiates, par le biais desquelles ils sauraient détecter, en plus des symboliques collectives, des messages immédiats - un geste, un mouvement, un son, une parole personnels ou personnalisés, et, ainsi, accéder à une nouvelle gamme d'informations.

La première affirmation de « $B$ », selon laquelle les séparations compliquent les mariages, est reçue de la part de « $A$ », sans résistance, même sans interrogation ; autrement dit, l'information émise est reçue par un agent dont le cadre de réception semble s'apparenter à celui de l'émettrice. Les notions évoquées sont très certainement partagées par les deux interlocuteurs, mais elles pourraient aussi intervenir semblablement dans une discussion entre d'autres agents ; c'est en ce sens que les individus puisent dans un répertoire dont ils disposent mais qui ne leur appartient pas en propre. L'histoire de leur société produit des situations maritales et familiales dont il est hautement probable qu'elles appartiennent à l'environnement de personnes spécifiques et qu'elles laissent en eux des impressions similaires. Or, dans la partie suivante de la séquence, où « $\mathrm{B}$ » rapporte les paroles d'une connaissance qui dit vouloir se marier loin de ses proches - autre notion admise implicitement et qui relève d'un arrière-fond - on sent chez " $A$ » que, malgré sa compréhension initiale des idées véhiculées, des précisions s'imposent; ce qui transparait dans sa première réplique - "Oué ? Ah oué... » - et aussi dans sa seconde, où il pose directement la question : " $\mathrm{Ah}$, pour le monde que tu vas inviter puis... ? ». $\mathrm{Si}$ « A » se montre d'abord en accord, c'est qu'il croit avoir compris en quoi le mariage est compliqué ; il préférera, néanmoins, vérifier auprès de « $\mathrm{B}$ » en la questionnant sur ce qu'elle entend, au juste, lorsqu'elle utilise le terme. Cela porte à croire que les catégories médiates disponibles ne témoignent pas seulement d'une conception symbolique générale, mais qu'elles contiennent également des particularités propres à des agents, bien sûr, et sans doute aussi à des histoires collectives spécifiques, et donc à une 
historicité non spécifique à l'échange. « $\mathrm{B}$ » répond à cette question par l'affirmative, après quoi « $A$ » accepte l'explication en admettant ne pas y avoir songé auparavant. « $\mathrm{B}$ » reprend - «Oué... cet aspect là, euh... » - et questionne brièvement l'idée soulevée par $\langle A$ $A$ » $\langle\mathrm{B} »$ reprend son idée initiale : « $[. .$.$] il reste que c'est quand même 'awkward' [. .$.$] », ce que « A$ » reconnait. De la notion de " compliqué », nous sommes passés à celle d'«étrange », délimitant donc davantage la symbolique utilisée et permettant à l'échange de suivre son cours, les interlocuteurs ayant maintenant la conviction de faire usage de référents analogues. Les propos qui suivent consistent essentiellement en un récit vécu de « $\mathrm{B}$ » où « $A$ » intervient à quelques reprises pour attester, encore une fois, de la validité des sentiments de « $B$ » et, par conséquent, de leur appartenance à une même collectivité, c'est-à-dire de leur usage de catégories médiates communes.

\section{Conclusion}

Nous avons mis à l'épreuve la proposition d'une approche rationalisante qui veut que si le discours n'est qu'intention, il n'est que rationnel. Nous l'avons fait de six façons dans le but aussi bien d'en tester la validité empirique que de la confronter aux principes d'une approche relationnelle.

L'analyse a révélé, dans un premier temps, que les propos que s'échangent les interlocuteurs dépendent davantage d'un effet d'ajustement ou de complémentarité que d'une intention quelconque. Elle a mis en évidence des effets d'interactivité. Elle a montré que l'interaction est déterminante de ce qui est dit. Ces résultats ne permettent certainement pas de nier que l'humain est capable d'intention - ce n'était d'ailleurs pas notre intention de le faire - ; ils permettent toutefois de remettre en question la thèse de l'universalité de l'intention, d'en relativiser la portée et ils invitent très certainement à une nouvelle modélisation du social, et même de l'action sociale.

Dans un deuxième temps, l'analyse a montré que l'émotion était inhérente aux échanges. Nous l'avons, en effet, toujours repérée, soit directement, soit indirectement. Elle est apparue presque intrinsèque aux réactions grâce auxquelles les interlocuteurs peuvent construire un dialogue. Cette animation des échanges par l'émotion atténue à nouveau la thèse d'un acteur fondamentalement intentionnel. Certes, elle relativise en même temps la thèse d'un acteur rationnel, non pas en annulant le fait de la rationalité humaine, mais plutôt en mettant en évidence le caractère partiel de cette rationalité dans l'agir et en déposant cet agir davantage dans la communication que dans la subjectivité. 
Dans un troisième temps, l'analyse a indiqué que l'émotion n'avait pas une intention à sa source et certainement pas une intention qui eût précédé l'échange. Même dans les cas où l'on aurait pu attribuer le sens des propos à une quelconque intention qui serait survenue au cours de l'échange, cette intention était volatile. Jamais le discours n'a suivi de trame prédéfinie dont le sens se fût compris par référence à l'orientation que lui eût donnée un acteur. Comment, quand on y pense vraiment, une émotion peut-elle être le produit d'une intention? Il faudrait que ce soit alors un jeu. On peut imaginer que le fait d'éprouver quelque chose puisse faire naitre une intention, mais comment peut-on concevoir que ce qu'il adviendra de cette aspiration sera la simple réalisation de la projection d'un sujet agissant en dehors de toute relation?

Dans un quatrième temps, la question se posait de savoir si l'émotion contient une part de socialité. L'analyse a ici, à nouveau, été éloquente. Après avoir déterminé que les propos sont fonction de réactions, que ces réactions sont partiellement émotives et que l'émotion ne peut s'expliquer à partir de l'intention, restait à découvrir si l'émotion est possible en dehors de la socialité, ce que, par ailleurs, la plupart des travaux sérieux en sciences sociales empêchaient de croire malgré l'insistance des théories de l'action. Une approche relationnelle veut que l'humain soit toujours à la fois rationnel et émotif, et que cette dualité de sa psyché ne soit possible que par sa socialité : sans inscription dans le symbolique, il y a difficilement vécu et donc émorationalité ; l'ordre symbolique suppose des vécus particuliers, mais il est inconcevable au niveau de la simple subjectivité ; sans ordre symbolique, sans relations humaines, il n'y a ni compréhension, ni intercompréhension de ce qui est vécu. Les agents ne se comprennent que dans la socialité ; ils ne se comprennent ni en fonction d'un seul intérêt, ni en fonction d'une simple intention.

Mais l'influence du symbolique sur l'acteur et de l'acteur sur le symbolique n'est possible que de façon historique. Cette dialectique suppose une socialité toujours en train de se faire. L'émotion suppose la socialité, mais elle suppose tout autant l'historicité. C'est bien ce que rappelle l'analyse qui a été faite dans un cinquième temps quand elle découvre que l'échange est lui-même histoire en train de se faire, quand elle indique que l'émotion est produite par cette histoire et qu'elle produit en retour cette même histoire, bien arrimée qu'elle est à la raison. Comprendre, dans les relations humaines, c'est, certes, disposer logiquement de l'information, mais c'est aussi se laisser émouvoir. Nous avons observé de plusieurs façons les signes d'une historicité spécifique : dans la particularité des propos qui se développaient, dans la complicité qui prenait forme entre les agents 
communicants, dans la dépendance des humeurs à l'égard des interlocuteurs et de leur discours. Ce caractère historique des relations humaines, de l'émotion, l'analyse qui a été faite dans un sixième temps l'a bien signalé, elle aussi, quand elle a poussé le regard vers la dimension plus large de l'histoire. Une histoire spécifique n'est possible que parce qu'une histoire plus générale est en cours.

Dans ce travail, nous avons essayé de soumettre à l'épreuve de l'empirie les principes d'une théorie de l'action ou d'une sociologie centrée sur l'acteur. Cet essai est aussi modeste que nécessaire. Il est modeste, car il est minuscule à côté de tout ce qui reste à faire et surtout à cause du caractère innombrable et imposant des travaux qu'il remet en question. Il est nécessaire parce que, à nos yeux, les théories de l'action ne nous semblent pas à même de rendre compte de la complexité des relations humaines et c'est la raison pour laquelle nous privilégions une approche davantage centrée sur la relation, sur la communication humaine, que sur l'action. Mais une telle théorie est à faire. Les catégories critiques qu'elle emploie émoraison, communication, historicité, socialité, relation, - ne représentent qu'un environnement qui favorise la dénonciation et qui commande désormais une nouvelle sociologie qui génèrera des catégories plus analytiques. Et c'est à cette tâche qu'il nous faudra nous adonner sous peu. En attendant, il nous semble nécessaire de consolider la critique à l'égard des théories de l'action en répondant aux arguments que les porte-parole de ces théories ne manqueront pas de faire valoir contre notre travail.

Certains suggéreront que le thème que nous avons retenu pour animer les échanges a pu biaiser les résultats de l'analyse. Le thème du mariage, en effet, peut facilement faire intervenir l'émotion. Dans leur esprit, la présence ou l'absence de l'émotion serait facteur de ce dont on parle plutôt que des relations humaines en elles-mêmes. Il faudra le vérifier en comparant des échanges en fonction de diverses thématiques.

Certains, encore, mettront en doute les circonstances selon lesquelles ont eu lieu les échanges. Ils pointeront du doigt le caractère artificiel des rencontres, c'est-à-dire le fait que les conditions matérielles de l'échange aient été provoquées par le devis de recherche. Il faudra vérifier si l'on arrive à des résultats semblables quand la communication humaine a lieu dans des conditions qui lui sont usuelles.

D'autres soulèveront la question de la taille des groupes. Ils avanceront alors que les résultats des analyses auraient été moins probants s'ils avaient été tirés d'ensembles plus grands que ne le sont les dyades. Il faudra alors vérifier si les préceptes de la théorie de l'action dépendent de la taille des groupes. 
D'autres, encore, invoqueront la question de la culture, croyant qu'il aurait pu en être autrement si, par exemple, l'échantillon n'était pas francoontarien, canadien, nord-américain, occidental. Ce sera là une autre hypothèse à vérifier.

D'autres, enfin, s'en prendront à la structure de l'échange en soutenant que si les agents communicants étaient placés devant des enjeux réels, et contraints d'en venir à des décisions, comme c'est le cas, par exemple, dans bon nombre de comités, nous eussions alors observé les phénomènes qui sont prédits par les théories de l'action. C'est à nouveau là une vérification qu'il faudra faire.

Mais on notera que la plupart de ces réserves qu'on nous fera entendre, et à bon droit, obligent désormais à procéder à des analyses pour lesquelles le caractère relatif de l'intention est acquis.

On ne peut réellement dépasser une théorie simplement en opposant de nouvelles définitions aux siennes. En ce sens-là, la simple mise en évidence de la notion d'émoraison ne constitue pas en elle-même un dépassement de la notion de raison quand il s'agit de caractériser l'acteur social. Nous en sommes bien conscients. La notion d'émoraison, ici, ne nous a servi qu'à faire valoir le caractère obsolète d'une analyse de type rationalisante ou phénoménologique. Elle importe moins par ce qu'elle désigne que par l'obligation dans laquelle elle met désormais le chercheur d'aborder l'action humaine non pas à partir de sa subjectivité, mais à partir des relations dans lesquelles il s'inscrit et sans lesquelles, de toute façon, il n'y a pas subjectivité. La mise en évidence de la notion d'émoraison, l'insistance pour une approche relationnelle, ne servent ici qu'à ouvrir une voie, qu'à entamer la construction d'un appareillage conceptuel qui servira à voir au-delà de la lunette phénoménologique. Mais ni la conceptualisation proposée, ni l'approche envisagée ne prétendent à l'exclusivité : d'autres manières de faire, en effet, et peut-être plus fécondes, peuvent être imaginées. On peut néanmoins penser que de tels projets viendront à mieux servir l'analyse des relations humaines un peu comme la notion de raison dialectique a permis plus tôt d'entrevoir que la notion de raison analytique était loin de pouvoir contenir en elle l'ensemble des manières dont un humain non seulement réfléchit son monde, mais aussi le vit, et le vit aussi bien en faisant qu'en subissant son histoire. 


\section{Références}

Alexander, Jeffrey, Theoretical L ogic in Sociology, vol. 1-4, Berkeley, University of California Press, 1982-1983.

Berthelot, Jean-Michel, " Le devoir d'inventaire », in Philippe Cabin (dir.), La sociologie. Histoires et idées, Auxerre, Sciences humaines, 2000, p. 250-251.

Blau, Peter, Exchange and Power in Social Life, New York, Wiley, 1964.

Bouchard, Pierre, Contribution à la critique de la rationalité utilitaire. Pour un modele de remplacement des théories de l'action humaine, mémoire de maitrise, Sudbury, Université I aurentienne, 2000.

Boudon, Raymond, I a place du désordre. Critique des théories du changement, Paris, PUF, [1984] 1991.

Bourdieu, Pierre, L a distinction : critique sociale du jugement, Paris, Minuit, 1979.

Caillé, Alain, Don, intérêt et désintéressement. Bourdieu, Mauss, Platon et quelques autres, Paris, La Découverte/M.A.U.S.S., 1994.

Caillé, Alain, Critique de la raison utilitaire. Manifeste du MAUSS, Paris, La Découverte, 1989.

Crozier, Michel et Erhard Friedberg, L'acteur et le systeme, Paris, Seuil, Point politique, 1977.

Durkheim, Émile, Ie suicide : étude de sociologie, Paris, PUF, [1897] 1969.

Durkheim, Émile, Les règles de la métbode sociologique, Paris, PUF, [1894] 1937.

Foucault, Michel, L'archéologie du savoir, Paris, Gallimard, Bibliothèque des sciences sociales, 1969.

Foucault, Michel, Les mots et les choses. Une archéologie des sciences bumaines, Paris, Gallimard, Bibliothèque des sciences humaines, 1966.

Friedman, Debra et Michael Hechter, «The Contribution of Rational Choice Theory to Macrosociological Research ", Sociological Theory, n" 6, 1988, p. 201-218.

Garfinkel, Harold, Studies in Ethnomethodology, Englewood Cliffs, N.J., Prentice-Hall, 1967.

Girard, Mélanie, Relations bumaines et production dinformation : l'échange comme objet d'étude d'une approche relationnelle, mémoire de maitrise, Sudbury, Université Laurentienne, 2004.

Godbout, Jacques T. et Alain Caillé, L’esprit du don, Montréal, Boréal / Paris, La Découverte, 1992.

Goffman, Erving, The Presentation of Self in Everyday Life, Woodstock, New York, Overlook Press, 1973.

Habermas, Jürgen, Le discours philosopbique de la modernité. Douze conférences, traduit de l'allemand par Christian Bouchindhomme et Rainer Rochlitz, Paris, Gallimard, Bibliothèque de philosophie, [1985] 1988.

Habermas, Jürgen, Théorie de l'agir communicationnt, tomes I et II, traduit de l'allemand par Jean-Louis Schlegel, Paris, Fayard, L'espace du politique, [1981] 1987. 
148 NPSS, VOLUME 1, NUMÉRO 2, 2006

Homans, George C., Social Behavior: Its Elementary Forms, New York, Hartcourt, Brace and World, 1961.

I aflamme, Simon, Communication et émotion. Essai de microsociologie relationnelle, Paris, L'Harmattan, 1995.

Luhmann, Niklas, Social Systems, traduit de l'allemand par John Bednarz et Dirk Baecker, Stanford, Cal., Stanford University Press, [1984] 1995.

Morin, Edgar, La méthode, tomes 1-6, Paris, Seuil, 1977-2004.

Morin, Edgar, Le paradigme perdu, la nature bumaine, Paris, Seuil, 1973.

Quéré, Louis, "Langage de l'action et questionnement sociologique », in Paul Ladrière, Patrick Pharo et Louis Quéré (dir.), La théorie de l'action. Le sujet pratique en débat, Paris, CNRS, 1993, p. 53-83.

Sacks, Harvey, Lectures on Conversation, Oxford, Blackwell, 1995.

Smith, Dorothy, The Conceptual Practices of Power: A Feminist Sociology of Knowledge, Toronto, University of Toronto Press, 1990.

Touraine, Alain, Critique de la modernité, Paris, Fayard, 1992.

Touraine, Alain, Le retour de l'acteur : essai de sociologie, Paris, Fayard, 1984.

White, Harrison, Identity and Control: A Structural Theory of Social Action, Princeton, N.J., Princeton University Press, 1992. 